\title{
Determination of sodium fatty acid in soap Formulation Using Fourier Transform Infrared (FTIR) spectroscopy and multivariate calibrations.
}

\begin{abstract}
Fourier Transform Infrared (FTIR) spectroscopy using an attenuated total reflectance (ATR) accessory has been investigated as a method for the determination of sodium-fatty acid (sodium-FA) in soap formulations. Multivariate calibrations namely partial least squares regression (PLS) and principle component regression (PCR) were developed for the prediction of sodium-FA using spectral ranges on the basis of relevant IR absorption bands related to sodium-FA. The sodium-FA content in soap formulations was predicted accurately at wavenumbers of 1,570-1,550 $\mathrm{cm}-1$, which is specific for RCOO- Na+ vibration. The PLS method was found to be a consistently better predictor when both PLS and principal component regression (PCR) analyses were used for quantification of sodium-FA. Furthermore, FTIR spectroscopy can be an alternative technique to American oil Chemist Society methods which use a titrimetric technique because FTIR offers rapid, easy sample preparation and is friendly to the environment.
\end{abstract}

Keyword: FTIR spectroscopy; Partial least squares regression; Principle component regression; Soap formulation; Sodium-fatty acid. 